\title{
Size Controlled Synthesis Of PHB Microparticles
}

\author{
Ramkumarpandian S, Sundar K
}

\begin{abstract}
Polymer microparticles are being widely considered for delivery of drugs during therapies, by stabilizing and ensuring biological activity, which facilitates adsorption and delivery of these drugs to the target site. In the present study, size formulation of $\mathrm{PHB}$ (polyhydroxybutyrate) microparticles was performed using a 3-factor 2-level Response Surface Methodology (RSM) design. The results were analyzed by DLS measurements. Experimental responses of a total of 20 formulations resulted in various particle sizes ranging between $100 \mathrm{~nm}$ to $\sim 8000 \mu \mathrm{m}$. These size controlled microparticles can be utilized for various therapeutic applications.
\end{abstract}

\section{Keywords: PHB microparticles, RSM, DLS}

\section{INTRODUCTION}

Nanotechnology is sought-after field employed with various applications in fiber, textile, agriculture, electronics, forensics, space and medical therapeutics [1]. Various nanomaterials are being proven for their role in targeted drug delivery[2]. Instead, microsized PHAs (Polyhydroxyalcanoates) are used as vectors for the controlled release of various drugs and the products of PHA degradation including monomers and oligomers are not harmful to the surrounding tissues [3]. Polymeric nanoparticles, called as "artificial viruses" [4] are biodegradable and biocompatible, fashioned by biological method [5] and have been applied for bio separation, sensing, molecular imaging and drug delivery by facilitating absorption of the corresponding materials and delivering them to the target site [6]. PHB microparticles with their various proven efficiencies can easily penetrate in to different tissues and can even pass through the blood-brain barrier by surface modification. For intravenous delivery, nanoparticles are superior to the micro particles. The smallest capillaries in the human body are 5-6 $\mu \mathrm{m}$ in diameter, which requires particles much smaller than its size for easy transverse through the capillaries that cannot be furnished by the micro particles and also prevent them from forming embolism [7]. Application of biopolymer microparticles in delivery of drugs diminishes the expenses and risks of toxicity [1]. Encapsulation of drugs with polymer microparticles increases the efficacy, specificity and tolerability of the not revise any of the drug. They are been proven for their

Revised Manuscript Received on December 05, 2019

* Correspondence Author

Ramkumarpandian $\mathbf{S}^{*}$, Department of Biotechnology, Kalasalingam Academy of Research and Education, Krishnankoil-626126

Sundar K, Department of Biotechnology, Kalasalingam Academy of Research and Education, Krishnankoil-626126. efficiency in gene therapy treating breast cancer cells, resulting in anti-proliferative effects [8]. Drug degradation and drug release can be managed by drug-to-polymer ratio; molecular weight and size of the polymer microparticles [2] The surface coatings of the polymer microparticles also influence the targeting characteristics of the microparticles. Since microparticles come into direct contact with the cellular membranes, penetration across the physiological drug barriers and the targeting capabilities depend upon the size and distributions of polymer microparticles [9]. Optimization of size distribution can be carried out using the statistical design, Response surface methodology [10]. Response surface methodology (RSM) is a statistical technique can be used to evaluate the relative significance of several factors, such as optimizing the PHB concentration, acetone concentration and agitation speed. Hence, it is comfortable to develop nanomaterials with précised factors. In the present study, PHB microparticles of various sizes were formulated by optimizing PHB concentration, acetone concentration and agitation using RSM and analysed for their effect over the size. The synthesized PHB microparticle of various sizes, by response surface methodology was characterized using particle size analyzer. On a commercial scale, this is the first report elucidating the optimization of size controlled synthesis of polymer microparticles which can be applied for distinctive functions in drug delivery, gene therapy and bioimaging based on their size specificity.

\section{MATERIALS AND METHODS}

\section{Materials}

All the materials purchased from Hi Media unless otherwise stated. PHB was synthesized from our laboratory [13].

\section{Preparation of microparticles}

The production of PHB and the synthesis of PHB microparticles were performed as previously reported [13, 14].

\section{Experimental design}

Design Expert 7.1.6 software (Stat-Ease, Inc., Minneapolis, trial version) was used. The central values for one factor at a time was mentioned in Table 1 [15].

Optimization of experimental data analysis and model validation

Central composite rotary design with two level three factor design was used to optimize the medium. The variables used were PHB, acetone concentration and agitation at five coded levels. Response studied was size of PHB microparticles

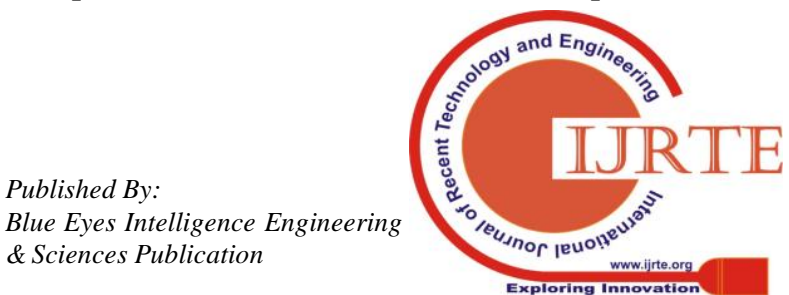




\section{Size Controlled Synthesis Of PHB Microparticles}

using a particle size analyzer and three dimensional graphs were fabricated to recognize the interaction of involved factors.

\section{Particle size analysis}

Particle-size distribution analysis was carried out after the production of PHB microparticles. Following the synthesis using solvent displacement method the solution was centrifuged at $\quad 16,400 \mathrm{rpm}$ for $30 \mathrm{mins}$ and the supernatant was removed. The pellet was resuspended with the phosphate buffer $\mathrm{pH}$ 7.0. The homogenous suspension of PHB-NPs was diluted 5-fold for all experiments involving measurement of DLS.

\section{Fluorescence effect of polymer microparticles}

The polymer dissolved in acetone was mixed with Nile red dye $(1 \% \mathrm{v} / \mathrm{v})$ and incubated at $37^{\circ} \mathrm{C}$ for $5 \mathrm{~min}$. The microparticles were synthesized by the micro precipitation method. Afterward, the solution was centrifuged at 16,400 rpm for $30 \mathrm{~min}$ to remove the supernatant and unbound Nile red. The pellet obtained was resuspended in the phosphate buffer $\mathrm{pH}$ 7. Fluorescence microscopy were employed to analyse samples under fluorescence microscopy.

\section{RESULTS}

\section{Synthesis of PHB microparticles}

Solvent displacement technique was used to synthesize PHB microoparticles. Acetone containing PHB was injected drop-by-drop into aqueous solution which resulted in the formation of the microparticles. TEM is utilized to analyse nanoparticles [14] After confirming the synthesis of the microparticles, the size of the microparticles were optimized using response surface methodology and the results were evaluated by DLS measurements.

\section{Response surface methodology}

Dynamic light scattering method was utilized to analyse the size of the nanoparticles.

$\mathrm{Y}($ Size of PHB NP $)=+8489.42-64.61 * \mathrm{~A}+83.15 *$

$\mathrm{B}-511.60 * \mathrm{C}+65.00 * \mathrm{~A} * \mathrm{~B}+13.25 * \mathrm{~A} *$

C-20.25* B

$* \mathrm{C}-3009.25 * \mathrm{~A} 2-2834.95 * \mathrm{~B} 2-1899.63 *$

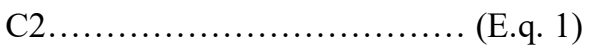

$\mathrm{Y}$ is the yield of the PHB microparticles size; $\mathrm{A}$ is acetone $(\mathrm{v} / \mathrm{v})$; B is PHB (mg/ mL) and C is agitation (rpm). The statistical significance of the model equation was evaluated by the F-test for analysis of variance (ANOVA), which showed that the regression is statistically significant at $99 \%$ $(\mathrm{p}<0.05)$ confidence level. $[16,17]$. RSM was employed to study the factors influencing nanoparticles. (Fig. 1). As a result particle sizes ranging from $\sim 100 \mathrm{~nm}$ to $\sim 8000 \mu \mathrm{m}$ were obtained with various conditions of the three factors optimized in the experiments. The mean average size of the particles obtained were about $300 \mathrm{~nm}$ for $1 \mathrm{mg}$ PHB in 3.68 $\mathrm{ml}$ acetone and $8400 \mathrm{~nm}$ for $1 \mathrm{mg}$ PHB in $2 \mathrm{ml}$ acetone (Fig. $2 \mathrm{~A})$. These results indicate that the size of the PHB microparticles can be modulated by directly changing the concentration of optimized factors, PHB and acetone.

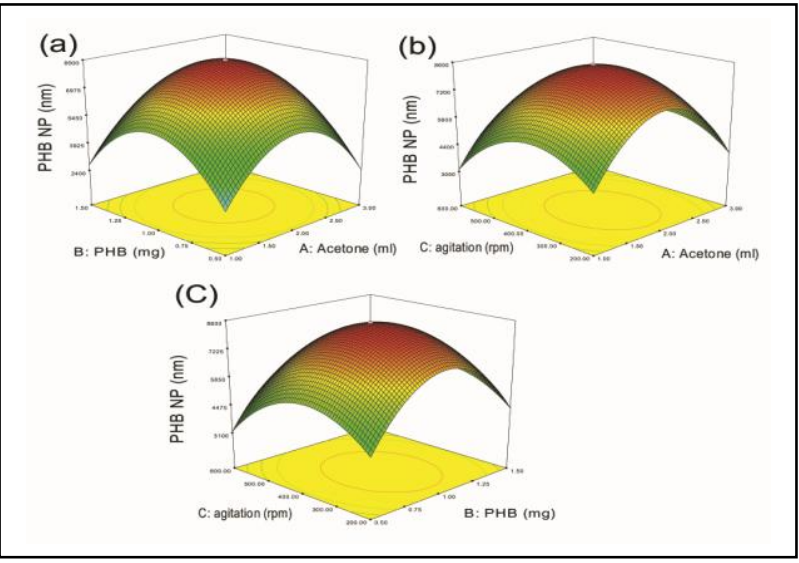

Fig. 1. Three-dimensional curve showing the interaction of various factors influencing the size of PHB

microparticles (a) PHB concentration and Acetone, (b) Agitation and Acetone, (c) Agitation and PHB concentration.

\section{Fluorescence of PHB microparticles}

Nile Red emits fluorescence when it binds to PHB-MPs. The PHB microparticles synthesized by the optimized factors were conjugated with Nile red dye, leading to the formation of fluorescent microparticles that were fixed on glass slide for fluorescence microscopy. Microparticles tagged with Nile Red were emitted fluorescence at $590 \mathrm{~nm}$ (Fig. 3A)

\section{DISCUSSION}

In this report, the factors influencing the size of the polymer microparticles were studied. Also the variations in the size of the microparticles with respect to the change in the conditional factors are being derived. To our knowledge, this is the first report elucidating the synthesis of PHB-NPs by varying the polymer concentration, acetone and agitation speed through response surface methodology. Wina and Feng, (2005) reported that biodegradable microparticles provide an alternative solution for anticancer oral drug delivery due to their small size [18]. Moreover, the natural occurrence of 3-hydroxy butyric acid in the blood, which is a PHB degraded product $[19,20]$ affirms the non-toxic nature of PHB during clinical treatments. The revelation relies on the fact that the PHB biopolymers used in the present study are produced only through biological means [13], whereas US Food and Drug Administration (FDA) approved polymers such as PLA and PLGA for human use undergo certain chemical processes to convert the monomeric units into the polymers [2]. Engineered polymers find their vivid application in developing advanced drug delivery systems and in molecular imaging [21]. Here, the polymer microparticles were produced by solvent displacement method that considered PHB, acetone concentration and agitation speed, where polyvinyl alcohol (PVA) was used as emulsifier. To study the variation in the size of polymer microparticles, central composite rotary design was performed. 


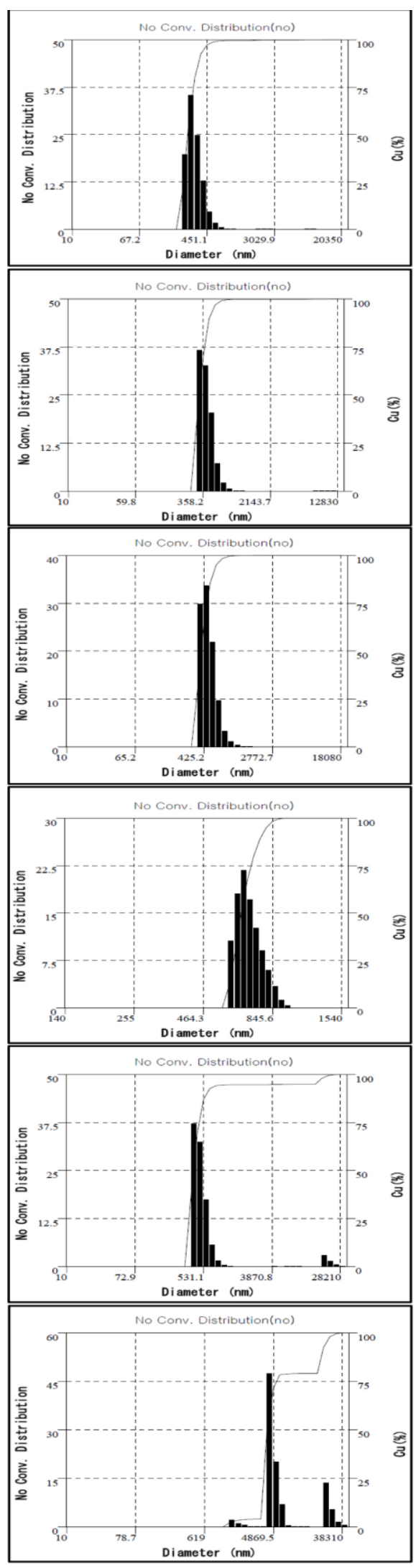

Fig.2. PHB microparticles synthesized using response surface methodology was analyzed by DLS.
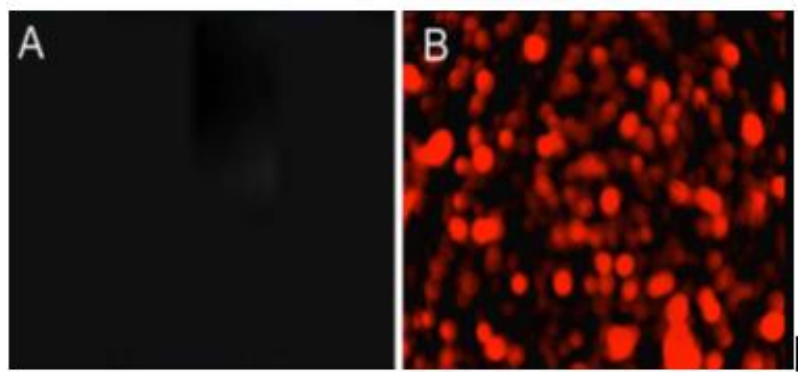

Fig. 3. Fluorescence image of the PHB microparticles Among three variables tested in this investigation, the concentration of PHB and volume of acetone influenced the size of the PHB microparticles. An increase in polymer concentration led to a significant increase in the size of nanoparticles produced, which is in accordance with results from the previous reports [22]. And the increase in the viscous resistance of the emulsion mixture was obtained in the organic phase, when the PHB concentration was increased. This in turn led to the reduction in shear stress resulting in droplets with larger size, through the absorption of the agitation energy [23]. In our experiments using the independent factors, the average size of the microparticles produced were $300 \mathrm{~nm}$ and $8400 \mu \mathrm{m}$ which was subsequently confirmed using particle size analyzer. Microparticles having size between 100 to $300 \mathrm{~nm}$ are attractive carriers because they can easily penetrate into deep tissue and are easily internalized by cells [24, 25]. Also, polymer nanoparticles ranging from 10 to $500 \mathrm{~nm}$ in size can selectively deliver the drug to tumor tissue [26, 27]. The hydrodermic radius of dispersed particles in aqueous phase was measured by DLS. Due to their microsize, polymer microparticles are known to possess a prolonged drug circulation time, drug stability, and escape from the reticuloendothelial system. A fluorescent micrograph was taken for Nile red bound PHB microparticles where the microparticles emitted fluorescence at $>590 \mathrm{~nm}$ and the observed result corroborates with prevailing evidences [28] which can provide a good means for the imaging studies.

\section{CONCLUSION}

In the current study, we optimized the size of polymer microparticles using response surface methodology by varying the $\mathrm{PHB}$, acetone concentration and agitation speed. Hence these PHB microparticles synthesized of various sizes at optimized conditions have a vivid futuristic application in the field of localized gene therapy in retinal disorders and cancer treatments when the DNA is encapsulated with PHB and also in bio imaging frames using quantum dots.

\section{REFERENCES}

1. Kumari, S. K. Yadav, S. C. Yadav, Colloids. Surf. B. 75 (1), 1-18 (2009).

2. H. Faraji, P. Wipf, Bioorg. Med. Chem. 17, 2950 -2962 (2009).

3. Y. C. Yao, X. Y. Zhan, J. Zhang, X. H. Zou, Z. H. Wang, Y. C. Xiong, J. Chen, G. Q. Chen, Biomaterials 29, 4823-4830 (2008).

4. H. Cohen, R. J. Levy, J. Gao, I. Fishbeinm, V. Kousaev, S. Sosnowski, S Slomkowski, G. Golomb, Gene Therapy 7, 1896-1905 (2000).

5. J. I. Choi, S. Y. Lee, Bioprocess. Biosyst. Eng. 17, 335-342 (1997).

6. B. Woitiski, F. Veiga, A. Ribeiro, R. Neufeld, Eur. J. Pharm. Biopharm. 73, 25-33 (2009). 


\section{Size Controlled Synthesis Of PHB Microparticles}

7. M. L. Hans, A. M. Lowman, Curr. Opin. Solid. St. M. 6, 319-327 (2002).

8. S. Prabha, V. Labhasetwar, Mol. Pharm. 1(3), 211- 9 (2004).

9. L. B. Peppas, J. O. Blanchette, Adv. Drug. Deliv. Rev. 56 (11), 1649-59 (2004).

10. V. Deepak, K. Kalishwaralal, S. Ramkumarpandian, S. V. Babu, S. R. Senthilkumar, G. Sangiliyandi, Bioresour. Technol. 99 , 8170-8174 (2008).

11. C. Montogomery, Design and Analysis of Experiments, 5th Edition, John Wiley and Sons, New York, 2001

12. R. H. Myers, D. C. Montogomery, Response Surface Methodology: Process and Product Optimization Using Designed Experiments, 2nd Edition, John Wiley and Sons, New York, 2002.

13. S. R. Pandian, V. Deepak, K. Kalishwaralal, J. Muniyandi, N. Rameshkumar, S. Gurunathan, Bioresour. Technol. 101, 705-711 (2010).

14. S. R. K. Pandian, V. Deepak, K. Kalishwaralal, J. Muniyandi, N. Rameshkumar, S. Gurunathan, Colloids. Surf. B. 74, 266-273 (2009).

15. J. L. Uma Maheswar Rao, T. Satyanarayana, Bioresour. Technol. 98(2), (2007) 345-352.

16. Chauhan, R. Gupta, Process. Biochem. 39, 2115-2122 (2004).

17. P. D. Haaland, Statistical problem solving, in: Experimental Design in Biotechnology, Marcel Dekker Incorporation, New York, 1989.

18. K. Y. Wina, S. S. Feng, Biomaterials 26, 2713-2722 (2005).

19. M. I. Wiggam, M. J. O'Kane, R. Harper, A. B. Atkinson, D. R. Hadden, E. R. Trimble, P. M. Bell, J. Biomed. Mater. Res. A. 46, 494-503 (1999).

20. M. Zinn, B. Witholtb, T. Eglia, Adv. Drug. Deliv. Rev. 53, 5-21 (2001).

21. J. H. Kim, K. Park, H. Y. Nam, S. Lee, K. Kim, I. C. Kwon, Prog. Polym. Sci. 32, 1031-1053 (2007).

22. X. Song, Y. Zhao, S. Hou, F. Xu, R. Zhao, J. He, Z. Cai, Y. Li, Q. Chen, Eur. J. Pharm. Biopharm. 69, 445-453 (2008).

23. R. Manchanda, A. Fernandez-Fernandez, A. Nagesetti, A. J. Mc Goron, Colloids. Surf. B. 75(1), 260-267 (2010).

24. S. D. Conner, S. L. Schmid, Nature, 422, 37-44 (2003)

25. K. A. Woodrow, Y. Cu, C. J. Booth, J. K. Saucier-Sawyer, M. J. Wood, W. M. Saltzman, Nature Materials 8, 526 - 533 (2009).

26. F. Yuan, M. Dellian, D. Fukumura, M. Leunig, D. A Berk, V. P. Torchilin, R. K. Jain, Cancer. Res. 55, 3752-3756 (1995).

27. S. Kim, J. H. Kim, O. Jeon, I. C. Kwonb, K. Park, Eur. J. Pharm. Biopharm. 71, 420-430 (2009).

28. R. A. Fletcher, J. A. Brazinb, M. E. Staymatesa, B. A. Benner Jr, J. G. Gillen, Talanta 76, 949-955 (2008).

\section{AUTHORS PROFILE}

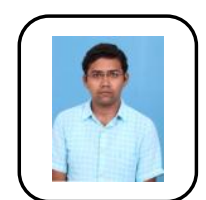

He was completed his Ph.D. degree in the field of Biotechnology in the year of 2016. At present he is working as assistant professor at the Department of Biotechnology, Kalasalingam Academy of Research and Education, Krishnankoil-626126.

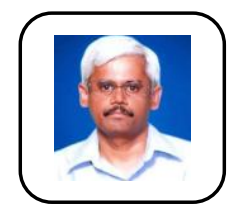

Dr Krishnan Sundar is currently working at Kalasaligam University as a Professor of Biotechnology and he is also the Dean of Planning and Development. Dr Sundar graduated from Madurai Kamaraj University with a doctoral degree in Microbiology and later carried out post-doctoral research in USA. In 2007 he returned to India as a faculty member of Kalasalingam University. He teaches Immunology and Vaccinology for Under Graduate students and Immunotechnology, Genomics and Proteomics and Infectious Diseases for Graduate Students. His major research interests are infection and immunity. 